\title{
RFID Antenna for UHF Band
}

\author{
Nicolae Crișan \\ Department of Communications \\ (Faculty of Electronics, Telecommunications and Information Technology) \\ Technical University of Cluj-Napoca, Romania \\ (nicolae.crisan@com.utcluj.ro)
}

\begin{abstract}
This paper presents a method to increase the detection range for RFID readers in the UHF radio band. The design is following especially the ISO 18000-6C standardization demands for the UHF band, operating from 865 to $868 \mathrm{MHz}$ in Europe and from 902 to $928 \mathrm{MHz}$ in North America. These regulations are allowing the RFID tags to operate near the metallic surfaces and in a wider range than its counterparts at lower frequencies. The paper highlights the advantages offered by the array antenna, especially by the beamforming to increase the reader surveillance zone. The paper describes some additional techniques that are allowing the usage of the circular polarization to increase the reader sensing range and to improuve detection. The design starts with simulation and optimization using HFSS and ends with the measurements that validate the simulation results.
\end{abstract}

\section{Keywords-UHF-RFID, HFSS, Circular polarization}

\section{INTRODUCTION}

The growth of the RFID sensors is significant, especially in the last decade. Even more popular, still in greater use, the UPC (Universal Product Codes) well known as barcodes have some significant shortcomings that can be overcome by using the RFID instead. The RFID tags don't need the line of sight and can cover more considerable distances from a few centimeters to 10 meters. In the market are some leading companies that had envisioned the possibility of using the potential of the UHF RFIDs in items traceability along the supply chain. Although, they are in use from 1991 when in the United States the first toll collection point on highways for vehicle access has been deployed. The biggest electronic library, Seattle Public Library [1], is enabling its UHF-RFID system, developed by TAGSYS, which is checking out the collection of books in real-time. The RFIDs are using the nearfield zone and coupling between the reader and the RFID antenna tag to exchange information. An UHF-tag has a passive integrated circuit that is switching on and off according to the inner ID code when the fields are strong enough to supply the device with the electric energy. The on\&off switching is varying the input impedance of the IC, thus modulating ASK (Amplitude Shift Keying) the backscattered signal. The idea is to gather enough energy from the reader carrier to rectify the signal and supply the IC with DC. In this case, the antenna is called rectenna, according to its role of RF to DC converter. At lower frequencies at 125 $\mathrm{KHz}$ and $13.56 \mathrm{MHz}$ (RFID/NFC - Radio frequency identification/ Near Field Communications), the antenna has a smaller gain due to its smaller size compared with the wavelength. The inductive antennas have narrow bandwidth and are tunable at a high-Q to meet the required size of the tag. These compromises could negatively change the antenna performance from the antenna gain point of view and spatial selection. Besides this, the coupling with other surrounding objects especially with metals makes the operation difficult near to impossible in the proximity of them. Other impairments are related to the mutual coupling between the tags that make the interrogation of the many, at once, difficult. These drawbacks clear the way for the next generation of the RFIDs in UHF near the microwaves range that allows the usage of the MIMO RFIDs and array antennas. In this case, the wavelength is closer to the size of the antenna which makes it more reliable from the detection point of view. Frequency increasing, often, is associated with gain and bandwidth improvement. The tuned antenna hasn't such a high-quality factor which makes the reading process more reliable due to the week couplings. The searching range increases up to a thousand times at the same antenna size. Outstanding results were encountered with the long rage readers at $866.5 \mathrm{MHz}$ by Murata Ltd., from Japan or by the FEIG Electronic GmbH from Germany. In this respect, one of the antenna leader manufacturer, Kathrein, has presented an innovative RFID antenna array on $866.5 \mathrm{MHz}$. The reader range built by Kathrein proposed an operational range up to 18 meters at only $50 \mathrm{~mW}(17 \mathrm{dBm})$. The higher the rage, the more antenna elements are necessary at the reader. Nevertheless, the growth in size leads to a UHF array that overcomes the HF antenna, in terms of sensitivity and gains at the same size. In this respect, the retro directive arrays together with the MIMO concept become more and more popular. The beamforming, the main achievement of the phased array antenna concept allowing a better spatial selection and identification not only a common range enlargement. This concept of a retro directive array could play an essential role in the RFID tag selection accuracy and reflect ability. This paper brings together some outstanding design ideas related to the RFID beamforming, not necessarily a new antenna design.

\section{ANTENNA ELEMENT DESIGN}

The circular microstrip antennas are part of the many designs that are following mainly the geometry of the tags. There are exciting designs in which the shortening circular patch has achieved better performance in terms of radiation efficiency and size. A cut in half antenna it doesn't lose much of the radiation gain but significantly reduce its aperture. This decreasing will allow the spacing necessary to bring closer the array elements and dropping mutual couplings as much as possible. The circular patch also has a comparable size with the dipole having a radius of about a quarter wavelength but a higher aperture. The cutting half-circular slit (etched in a semicircle), it reduces the size of the patch. The shorting to the ground is necessary to keep the resonant frequency centered 
to $800 \mathrm{MHz}$ and is undertaken by a slot disposed of, in parallel with the cut. The antenna becomes half in size and the fringing electric field changes accordingly to the new shape. The stray capacitance of the semicircular element is keeping the resonance between 850 and $950 \mathrm{MHz}$. A rectangular slot placed at the base of the cut forces the electric fields to rotate and make it circularly polarized. This is, in fact, a stronghold of this antenna to keep its efficiency to any polarization [2]. These traits make this antenna an excellent candidate for UHF RFID. The semicircular patch antenna due to its canceled half side experiences a weaker mutual coupling with the adjacent elements and remains suited for large array designs with a small aperture. The antenna was proposed first by Prof. N. C. Karmakar as it is presented at the RFID symposium held in Las Vegas in 2008 [3]. This approach uses the semicircular patch to decrease the coupling effect by increasing the space gap between the array elements. One element is operating upon three dielectric layers overlapped as those depicted in figure 1 . The first layer is a PCB with the relative electric permittivity $\varepsilon_{r 1}$. The active elements stand upon this layer. The active patch was highlighted as a perfect electric surface in HFSS. The second layer with the electric permittivity $\varepsilon_{r 2}$ has an active role in the beamwidth adjustment. In particular the thickness $H_{1}$ is one of the most sensitive parameters related to the antenna directivity. The paper proposes a modification that improves the antenna feeding by replacing the microstrip line used in [3] with a more efficient coplanar waveguide with the ground (CPWG - coplanar waveguide) in which the gaps and the linewidth, matching the characteristic impedance better. The coplanar waveguide configuration is most likely the best candidate for RFIDs due to low loss and the ability to concentrate the field inside the gap, so it is tapering easily the pin connected to the patch.

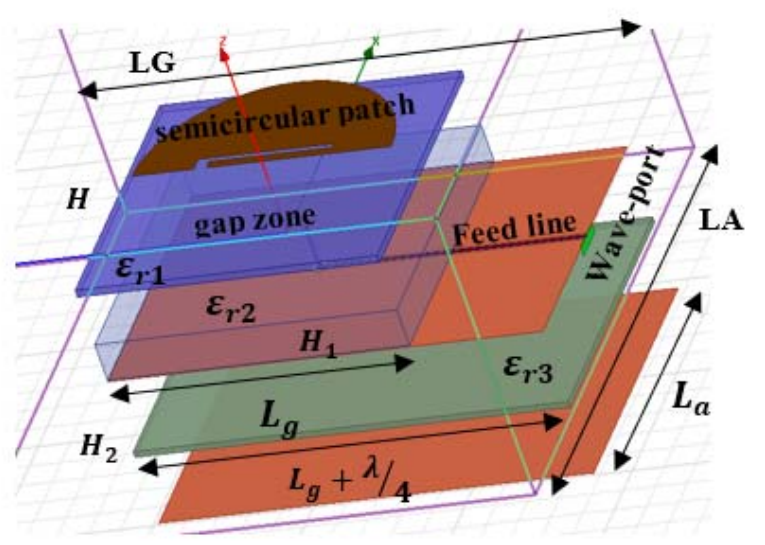

Fig. 1. Semicircular patch RFID antenna (sketch)

Another necessary adjustment taken into consideration here is the antenna resizing due to the effect of the second ground layer the wave-port is connected to. Compared to the antenna proposes in [3] and [4], the semicircular element introduced here has a smaller radius with about $10 \mathrm{~mm}$. The wave-ports in HFSS let open the possibility to use the deembedding operation to sniff out the impedance at a distance away from it. In this way, the antenna input impedance is extracted directly from the wave-port and will be displayed as it is just when it would be usually taken, directly from the feed point. In conclusion, one can have both impedance values from the ends of the CPWG line by reading the value from the wave-port directly with or without de-embedding option checked respectively. The third dielectric layer with the relative permittivity $\varepsilon_{r 3}$ plays a key role in the impedance adjustment and in the insertion loss mitigation. The width of the CPWG line corresponds to the characteristic impedance of 50 Ohms. This is a major advantage of this antenna that exhibits at its input the same impedance with the surge impedance of the transmission line. Thus, there is no need for an input impedance adapter which isn't lossless anyway. The mitigation of the loss has finally a positive effect on the RFID scanning range. The radius of the patch is about $81 \mathrm{~mm}$, resulting inside, a gap of $81 \mathrm{~mm}$ by discarding the semicircle cut. Although, the element geometry remains square of about $162 \times 162 \mathrm{~mm}$ to use the gap zone in mitigating the mutual couplings when an array with more elements is considered. The element antenna spacing embedded the gap zone inside the array aperture to keep the spacing between the patches. In Tab. 1 are all the parameters related to the antenna dimensions and size. fig. 1 depicted these parameters as well. All elements of the antenna from fig. 1 have been drawn in the HFSS editor by expanding apart the layers only to increase the sketch visibility (for a better view).

TABLE I. ANTENNA DimENSIONS, MATERIALS AND PARAMETERS

\begin{tabular}{|c|c|c|c|}
\hline PARAMETER & $\begin{array}{l}\text { LENGTH } \\
{[\mathrm{mm}]}\end{array}$ & PARAMETER & $\begin{array}{c}\text { LENGTH } \\
{[\mathrm{mm}]}\end{array}$ \\
\hline H & 1.6 & rad & 71 \\
\hline H1 & 25 & W & 2.5 \\
\hline H2 & 0.79 & ln & 72 \\
\hline$\varepsilon_{r 1}$ & 4.4 & wf & 7 \\
\hline$\varepsilon_{r 2}$ & 1.02 & hi & 8.5 \\
\hline$\varepsilon_{r 3}$ & 2.45 & ls & 65 \\
\hline$L_{g}$ & 189 & uv & 5 \\
\hline$L a$ & 189 & u1 & 29 \\
\hline$L A$ & 355.5 & xi & 9.5 \\
\hline$L G$ & 355.5 & - & - \\
\hline
\end{tabular}

In Fig. 2 are all parameters that are related to the antenna size and geometry. The tapering of the feeding pin and the CPWG line are highlighted. The CPWG line concentrates the fields in the gap that encircling the pin. Thus, the transition from the CPWG to the feeding pin exhibits a lower insertion loss.

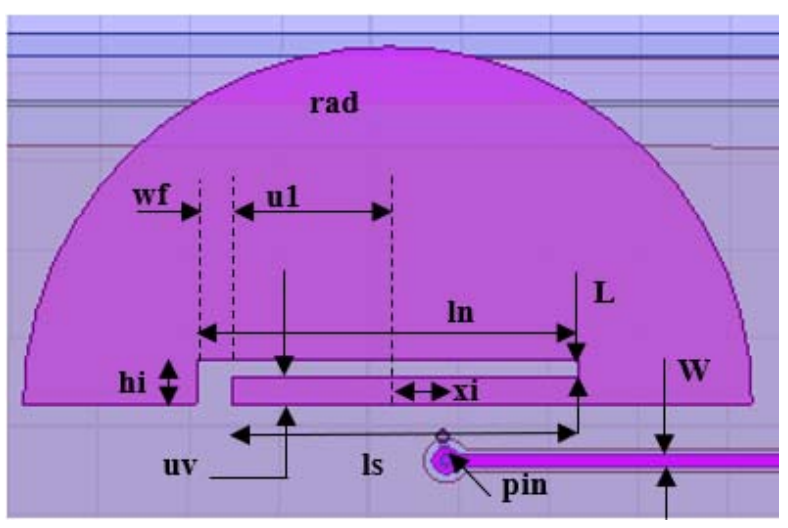

Fig. 2. Semicircular active patch (sketch) 
The feeding method proposed here in the paper is improving the overall bandwidth of the element. Also, the diameter of the pin has been decreased compared to the one proposed in [4] at $1 \mathrm{~mm}$. This shortening fits better the CPWG line transition to the pin at $50 \mathrm{Ohms}$ and improving the insertion loss with $0.1 \mathrm{~dB}$. The bottom layer is the CPWG feed layer that ends with a circular pad of about $6 \mathrm{~mm}$ in diameter. The material used for the bottom layer is Taconic TLX-0 with $\varepsilon_{r}=2.45$. The layer is very thin of about $0.79 \mathrm{~mm}$ to avoid the field fringing effect. This makes the feed line thinner at 50 Ohms and is allowing the strong couplings with the ground plane from the sides. The CPWG bottom ground it doesn't change the antenna resonance due to the upper layer ground. This second plane stands as a perfect electric boundary for both the patch and for the CPWG.

\section{SEMICIRCULAR RFID ANTENNA - SIMULATIONS AND MEASUREMENTS}

Simulations are made using the HFSS ver. 17.2 from Ansys Electronics Suite. The very first concern was about the feed line. A CPWG transmission line is replacing the microstrip line proposed in [3]. The CPWG line is being used more and more in RFID designs [5]. The design approach is improving the CPWG to pin transition. The CPWG's tap is increasing the antenna bandwidth (see fig. 3). The bandwidth is $116 \mathrm{MHz}(12.8 \%)$ and the best return loss at $882 \mathrm{MHz}$ is 29 $\mathrm{dB}$.

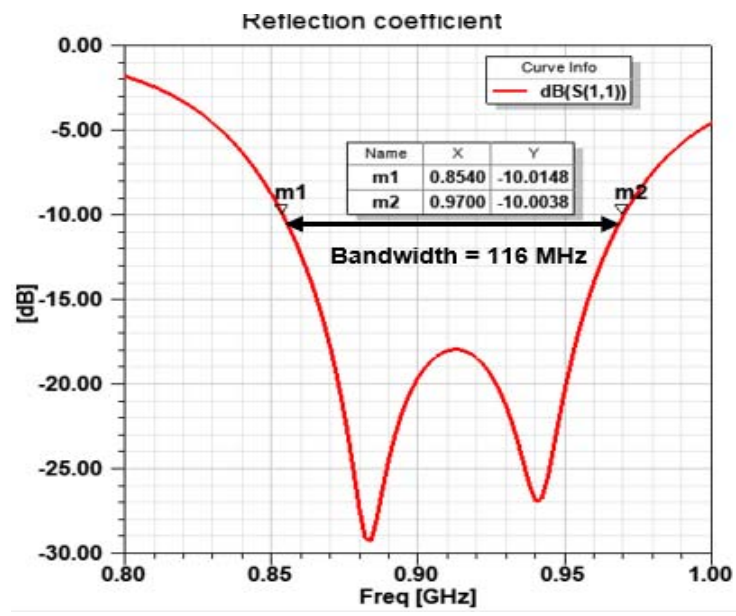

Fig. 3. The return loss of a single RFID element

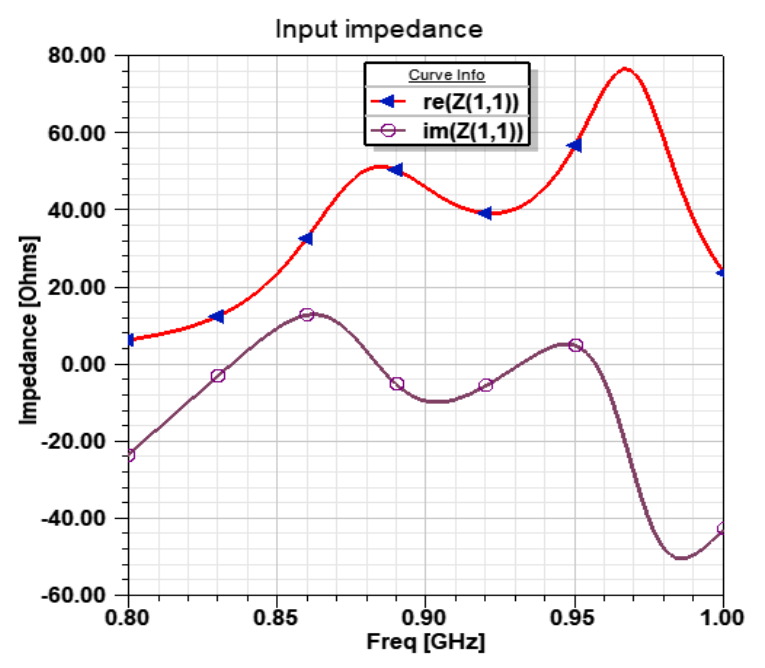

Fig. 4. The input impedance of a single RFID element
The bandwidth expands from the reported value in [3] with $20 \mathrm{MHz}$. With an overall bandwidth of $116 \mathrm{MHz}(13 \%)$ the proposed antenna could cover both North American and European standards. The bandwidth is consolidated by the second resonance depicted in fig. 4 at $930 \mathrm{MHz}$. The first resonance is encountered at $882 \mathrm{MHz}$. For both of them, the input impedance is about 50 Ohms. In fig. $4, Z_{11}$ is the input impedance at the wave port where the generator is connected. The real part is $\operatorname{re}\left(Z_{11}\right)$ as long as the imaginary part is abbreviated with $\operatorname{im}\left(Z_{11}\right)$. The gap around the pin line created at the end of the CPWG line where the line meets the pin (see Fig.2) concentrates the fields near the feed point. The feeding line, the gap, and the pin size have a great impact over the antenna bandwidth. The input impedance vs. the frequency is shown in fig. 4. As one can see, a thinner pin at the feeding point enables a stray capacitance that forces the second resonance at $931 \mathrm{MHz}$. The two resonances expand the bandwidth enlargement. This is sufficiently large to allow the usage of the antenna according to any designated radio bands in Europe (865-868 MHz) or in North America (902-928 $\mathrm{MHz}$ ) [6]. Another key aspect is related to the transmitted power of the RFID reader unit which is allowed up to $4 \mathrm{~W}$ e.i.r.p. This regulation is another issue that limits the scanning range regardless of the transmitted power.

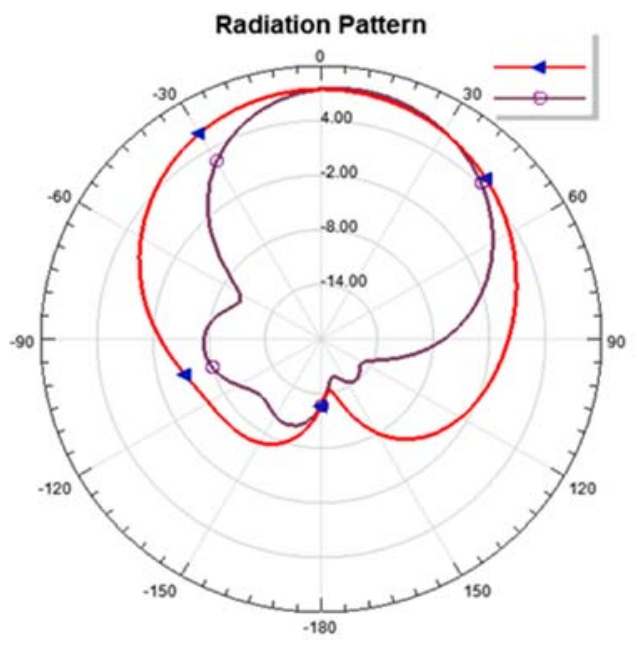

Fig. 5. The antenna radiation pattern of a single RFID semicircular patch, triangles mark the plot for an azimuthal angle of zero degrees and the circles for 90 degree

In the detection range enlargement, the antenna radiation pattern plays an important role. In U.S.A. the effective radiated power is limited to $4 \mathrm{~W}$. One single element has 7.69 $\mathrm{dBi}$ in terms of directivity along the z-axes when both azimuthal and elevator angles are zero. In fig. 5 two sections reveal the pattern for two orthogonal sections that stand for $P h i=0^{\circ}$ (marked with triangles) and for $P h i=90^{\circ}$ (circles). In fig. 6 is depicted the three-dimensional radiation pattern that stans for the total directivity as the one in two dimensions from fig.5. The main lobe has been segmented in three gray levels. The light gray region of the top bounds the radiation angle. As one can remark the antenna is not spatially selective, the radiation angle is larger than 60 degrees. The presented antenna is a good candidate for a UHF-RFID reader. It isn't suited for the use of tags being too large with its aperture of about $71 \mathrm{~mm}$. The high gain and its bandwidth recommend it for the reader unit. The next section is revealing the limitation a small directivity could have over the detection range anyway. All these results have been obtained in 
simulation approach with HFSS. The antenna implementation and measurements are evaluated in the next section. Although the radiation pattern is very hard to be evaluated without an anechoic chamber so fig. 5 and 6 results only through simulations in HFSS.

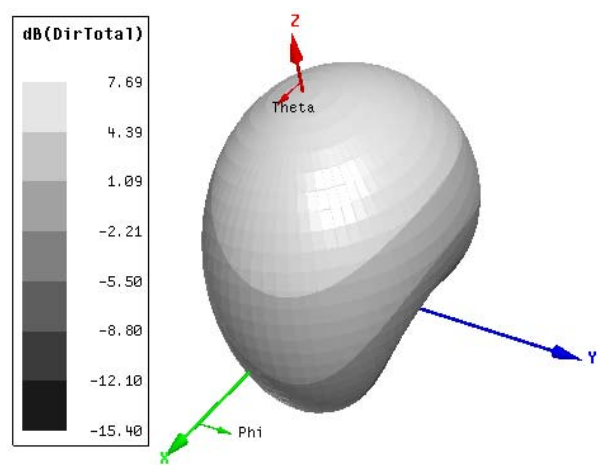

Fig. 6. A three-dimensional antenna radiation pattern of a single RFID semicircular patch

\section{ANTENNA MEASUREMENTS AND ThE SCANNING RANGE EXTENTION}

In fig. 6 the implementation of the UHF RFID antenna is depicted. The $71 \mathrm{~mm}$ radius of the antenna aperture is compared with a smaller coin of 10 bani (a tenth of a fraction from a Romanian leu). The $\mathrm{L}$ shaped slit is visible at the bottom held by the FR4 PCB. The fed point is asymmetrically placed but the feeding line is hidden between the layers.

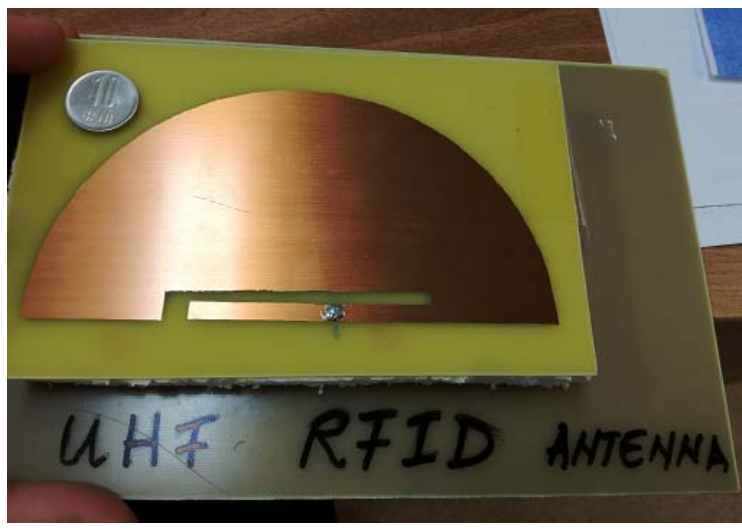

Fig. 7. Implementation of the UHF-RFID antenna using three dielectric layers

The CPVG line is also hidden by the polyurethane-foam (layer 2). Many estimation range techniques offer with great accuracy the scanning range for passive RFID tags. In this section, the RSS-based technique has been used also. This technique is the one chosen whenever the reader's sensitivity is taken as a reference to encircle the sensing range. The sensing area could be higher than the operational range depends on the signal processing and modulation technique involved in the process. The RSS-based technique is suited for any evaluation that hasn't consider a specific protocol or a given integrated circuits [7]. The main advantage is that the model is generally valid for many applications and protocols. Another stronghold of this model is related to the possibility to consider the tag positioning through the antenna 3D radiation pattern. In this approach [8] the backscatter power received by the reader is expressed as:

$$
P_{\text {backscattered }}=P_{T X} \eta G_{\text {tag }}^{2} G_{\text {reader }}^{2}\left(\frac{\lambda}{4 \pi d}\right)^{2 n},
$$

where:

- $\quad P_{T X}$ is the transmitted power (by the reader unit),

- $G_{t a g}$ is the tag antenna gain,

- $G_{\text {reader }}$ is the reader antenna gain,

- $\quad \eta$ is the power transfer efficiency of the passive tag,

- $\quad d$ is the distance.

The backscattered power decays with the distance but not as it normally does in free space with $d^{2 n}$. Integer $n$ is depending on the environment and could vary between 2 and 6 for indoors whenever it is not a line of sight in-between. For line of sight, $n$ is around 1.6 and 1.8. Ideal power transfer efficiency is 0.5 when the antenna impedance is conjugate matched to the integrated circuit chip input. In the conjugate matching circumstances, half of the received power is transferred to the chip and the rest is backscattered to the reader. When the efficiency $\eta$ is equal to unity there is no power transferred to the chip at all, all being backscattered to the RFID reader. The input impedance is measured with Vector Network Analyzer between 800 to $900 \mathrm{MHz}$.

Table II. Antenna measured parameters with the VNA

\begin{tabular}{|l|c|c|c|c|c|}
\hline \multirow{2}{*}{ Meas. } & \multicolumn{5}{|c|}{ UHF-RFID Antenna analyzed with the VNA } \\
\cline { 2 - 6 } & $\begin{array}{c}\text { Freq. } \\
{[\boldsymbol{M H} \boldsymbol{z}]}\end{array}$ & $\begin{array}{c}\text { Return } \\
\text { loss [dB] }\end{array}$ & $\boldsymbol{R}[\boldsymbol{\Omega}]$ & $\boldsymbol{X}[\boldsymbol{\Omega}]$ & $\boldsymbol{V S W R}$ \\
\hline $\begin{array}{l}\text { Lower } \\
\text { bound }\end{array}$ & 870.413 & 9.81 & 46.17 & -32.55 & 1.95 \\
\hline $\begin{array}{l}\text { Lower } \\
\text { rezonance }\end{array}$ & 895.265 & 25.20 & 54.45 & -3.62 & 1.11 \\
\hline $\begin{array}{l}\text { Higher } \\
\text { rezonance }\end{array}$ & 940.236 & 36.92 & 48.72 & -0.58 & 1.02 \\
\hline $\begin{array}{l}\text { Higher } \\
\text { bound }\end{array}$ & 966.271 & 9.89 & 41.23 & 29.39 & 1.94 \\
\hline
\end{tabular}

Lower bound and higher bound of the antenna bandwidth measured in Tab. II are in a good agreement with the simulations result from fig. 3 . The same agreement stands in favor of the two resonances at 895 and $940 \mathrm{MHz}$. In fig. 8 is plotted in Matlab the graph of equation 1, taking $G_{t a g}=$ $0 \mathrm{dBi}$ and $G_{\text {reader }}=7.7 \mathrm{dBi}$ from the direction pointing to the maximum of radiation (fig. 6).

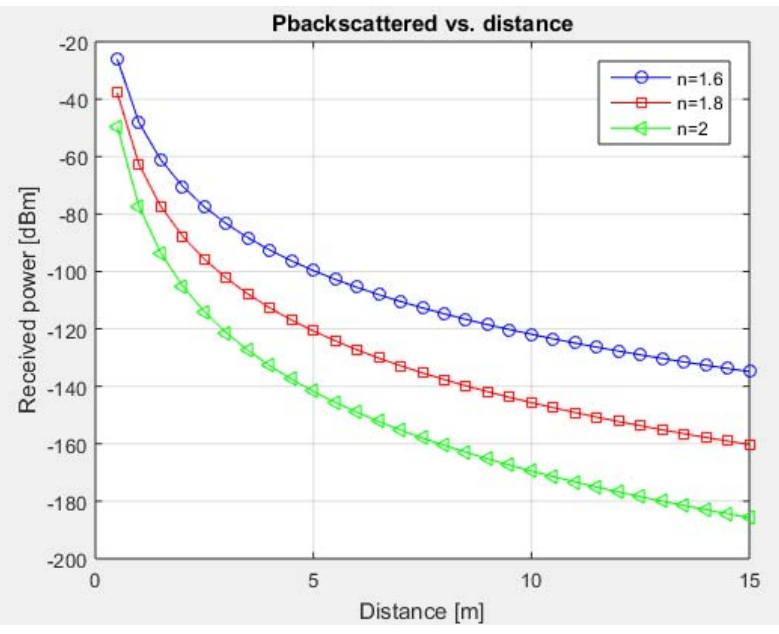

Fig. 8. Backscatered power versus distance for maximum effective radiated power (4 e.i.r.p or Ptx $=679 \mathrm{~mW}$ ) 


\section{RESULTS AND CONCLUSIONS}

Graphical representation of the equation 1 that is showing the backscatter signal received by the reader, reveals that the maximum range is around 10 meters regardless the gain of the reader antenna $\left(G_{\text {reader }}\right)$. The scanning range is limited mainly by the regulations that allowing only $4 \mathrm{~W}$ effective radiated power. Thereby the extension of the scanning range is not allowing through the transmitted power or antenna gain increasing at the reader. If we use a directive antenna with higher gain we have to lower the transmitted power at the reader radio interface. That is hoping to increase the detection range by increasing the antenna gain and reflectability at the tag. This will not lead to the power increase in the spectrum and could avoid and mitigate the interference with other tags. The regulation is following this rule that compels the players (readers and tags) not to play the game of the brut force as a basic strategy to sense a further away tag. That means that the most important is to increase the reflection efficiency of the tag antenna and its directivity. Another stronghold is the receiver sensitivity that could be count only if the designer can increase the isolation of the circulator device that makes the selection between the transmitted signal and the backscattered one. Anyway the presented antenna could be a good choice for the reader usage due to its smaller size and relative higher gain. This antenna will allow at least up to 5 meters when the line of sight is available between the reader and the tag. In very good circumstances when a very good sensitivity and isolation between the transmitted signal and the backscattered one is accomplished then the range can be increased up to 10 meters. The tag antenna must be smaller than the antenna presented here in the paper. In these circumstances here is harder to obtain a gain higher than $3 \mathrm{dBi}$ at the antenna tag. Nevertheless, a higher gain of the tag antenna could be possible if one can integrate two tag antennas and attached it as an array to a single chip. This will allow an increase in terms of directivity. Achieving only a $3 \mathrm{~dB}$ increase at the tag that will allow an extension of the scanning range at the reader with at least 10 meters for the same effective radiated power.

\section{REFERENCES}

[1] A. Shameen, "On Singapore Seeks Leading RFID Role," Phil. Trans. Roy. Soc. London, vol. A247, pp. 529-551, April 2006.

[2] S. M. Rathod, R. N. Awale and K. P. Ray "Shorted Circular Microstrip Antennas for $50 \Omega$ Microstrip Line Feed with Very Low Cross Polarization," Progress In Electromagnetics Research Letters, vol. 74, 2018, pp. 91-98.

[3] N. C. Karmakar, S. M. Roy, M. S. Iqram A, "Development of the smart antenna for EFID reader," IEEE RFID Symposium. Las Vegas, NV, April 2008.

[4] N. C. Karmakar, "Handbook of Smart Antennas for RFID System," Ed. John Wiley \& Sons., New Jersy, ISBN 978-0-470-38764-1, 2011.

[5] "Kathrein Introduces KRAI UHF SmartShelf Antenna" source Kathrein, in press news, RFID Solution Online Newsletters, March 2013.

[6] International Communications Union ITU-R, "Technical characteristics, standards, and frequency bands of operation for radiofrequency identification (RFID) and potential harmonization opportunities," Report ITU-R SM.2255-0, October 2012.

[7] F. Bouchereau, D. Brady, "Bounds on Range-Resolution Degradation Using RSSI Measurements,"IEEE International Conference on Communications,Vol.6,3246-3250, 2004.

[8] Edited by: M. Bolic, D. Simplot-Ryl, I. Stojmenovic, "RFID SYSTEM - Research Trends and Chalanges,"Willey, ISBN-978-0-470-74602-8, pag. 392, 2010 